\title{
Métodos de Aprendizaje para el Desarrollo de la Dimensión Estética en Estudiantes de Educación Inicial
}

\section{Learning Methods for the Development of the Aesthetic Dimension in Initial Education Students}

\author{
Esther Patricia Suaza-Atencio \\ esuaza72@yahoo.es \\ Institución Educativa San Mateo De Maganguè Bolívar. \\ Colombia \\ https://orcid.org/0000-0003-0119-9456
}

Recibido: 20 de noviembre de 2019

Aprobado: 15 de diciembre de 2019

\begin{abstract}
RESUMEN
En las últimas décadas los especialistas en las áreas sociales, aseguran que los programas que promueven el desarrollo de los niños son la mejor inversión para el fortalecimiento del progreso del capital humano que va a repercutir el crecimiento económico del país. Al interpretar esta situación, la presente investigación da cabida a la experiencia centrada en los estudiantes de educación inicial los cuales exigen la utilización de estrategias y métodos adecuados, en los que el aprendizaje se conciba cada vez más como resultado del vínculo entre lo afectivo, lo cognitivo, las interacciones sociales y la comunicación. El presente artículo ofrece elementos teóricos esenciales que permiten asumir posiciones fundamentadas acerca de los métodos de aprendizaje, lo que en ocasiones se aborda desde diversas posiciones, no siempre consistentes y con múltiples acepciones e interpretaciones. Asumiendo para ello el método descriptivo para dar detalles del fenómeno en estudio.
\end{abstract}

Descriptores: Métodos; Aprendizaje; Dimensión Estética; Desarrollo; Educación Inicial.

\section{ABSTRACT}

In the last decades, specialists in the social areas, ensure that programs that promote the development of children are the best investment for strengthening the progress of human capital that will impact the economic growth of the country. In interpreting this situation, the present investigation accommodates the experience focused on early education students which require the use of appropriate strategies and methods, in which learning is increasingly conceived as a result of the link 


\author{
CIENCIAMATRIA \\ Revista Interdisciplinaria de Humanidades, Educación, Ciencia y Tecnología \\ Año VI. Vol. VI. N${ }^{\circ}$. Edición Especial. 2020 \\ Hecho el depósito de ley: pp201602FA4721 \\ ISSN-L: 2542-3029; ISSN: 2610-802X \\ Universidad Nacional Experimental Francisco de Miranda (UNEFM). Santa Ana de Coro. Venezuela
}

\title{
Esther Patricia Suaza-Atencio
}

between the affective, the Cognitive, social interactions and communication. This article offers essential theoretical elements that allow us to assume informed positions about learning methods, which is sometimes approached from different positions, not always consistent and with multiple meanings and interpretations. Assuming for this the descriptive method to give details of the phenomenon under study.

Descriptors: Methods; Learning; Aesthetic dimension; Developing; Initial education.

\section{INTRODUCCIÓN}

En la actualidad, existen pocas dudas sobre la importancia del desarrollo infantil temprano, en el aprendizaje y en el posterior desarrollo social, destacando que las experiencias de los niños en sus primeros años son fundamentales para iniciar la vida y el aprendizaje con paso firme.

Asimismo, se reconoce la necesidad de una didáctica centrada en el sujeto que aprende, lo cual exige enfocar la enseñanza como un proceso de orientación del aprendizaje, donde se creen las condiciones para que los estudiantes no solo se apropien de los conocimientos, sino que desarrollen habilidades, formen valores y adquieran estrategias que les permitan actuar de forma independiente, comprometida ,creadora, para resolver los problemas a los que deberá enfrentarse en su futuro personal y profesional.

Todo ello conlleva la utilización de estrategias docentes y métodos que propicien un aprendizaje intencional, reflexivo, consciente y autorregulado, regido por objetivos y metas propias, como resultado del vínculo entre lo afectivo y lo cognitivo, además de las interacciones sociales y la comunicación, que tengan en cuenta la diversidad del estudiantado y las características de la generación presente en las aulas, con énfasis en la educación inicial y en las Tecnologías de Información y Comunicación. Lo anterior imprime a los estudiantes cierta autonomía en la búsqueda de información, lo cual no siempre es aprovechado por el docente por tanto, el proceso enseñanza-aprendizaje no se adecua a la realidad que impone el nuevo siglo. Desde el punto de vista educativo, es necesario, entonces, utilizar cada vez más en las 


\section{Esther Patricia Suaza-Atencio}

aulas herramientas propias de esta generación, para motivarlos y desarrollar sus habilidades a partir de sus estilos, modos y formas en que aprenden.

Sobre la base de lo planteado, la Organización para la Cooperación y el Desarrollo Económico OCDE (2017), el estudio demuestra que la inversión de un aprendizaje de alta calidad en la edad temprana, genera altas tasas de retorno en cuanto al rendimiento escolar y a una serie de resultados en la vida adulta, su ampliación de calidad es uno de los pasos más importantes que puede dar los países latinoamericanos para mejorar el desempeño general de la educación y mejorar la equidad social.

Las expectativas educativas del aprendizaje deben establecerse de forma clara para el desarrollo de los niños, debido a que proporciona perspectivas explícitas para el perfeccionamiento de una diversidad de competencias capaz de garantizar el cumplimiento efectivo de los derechos de cada uno de los niños y niñas. Reconocer a los niños y niñas como sujetos de derecho es, desde la perspectiva de la política pública, constituirlos como sujeto destinatario de un flujo regulado, robusto y constante de bienes, servicios y transferencias que generen, al interactuar, las condiciones de posibilidad para la realización de cada uno de los derechos que los estados se comprometieron a garantizar.

Es por ello que se perfila en el fortalecimiento de hacer realidad las posibilidades intelectuales, espirituales, afectivas, éticas y estéticas, que garantice el progreso de la condición humana, promoviendo el comportamiento del hombre consciente y capaz de ejercer el derecho al desarrollo justo, equitativo, que interactúe en convivencia con sus semejantes, con el mundo, que participe activamente en la preservación de los recursos naturales y culturales.

Lo antes planteado se puede consolidar con los actores que dinamizan el proceso educacional. Como principal se tiene al docente, que en su rol se proyecta en generar, promover el conocimiento, porque este es el instrumento por excelencia a través del cual se puede reflejar la realidad de un país, de la comunidad y su idiosincrasia. 


\section{Esther Patricia Suaza-Atencio}

En ese sentido, emergen las funciones del docente sustentado en el currículo; la educación preescolar ámbito de estudio de la investigación es el primer nivel de la educación formal y ordena la construcción de lineamientos generales en los procesos curriculares que constituyen las orientaciones para que las instituciones educativas ejerzan la autonomía, proyecten el trabajo permanente en torno al fortalecimiento de la calidad de la educación.

\section{DESARROLLO TEÓRICO}

La aproximación teórica de la presente, se orienta sobre la base del proceso de reflexión, análisis crítico y ajustes progresivos, donde el maestro factor clave para la investigación, hacen posible iniciar la indagación hacia nuevas realidades en donde imaginación de nuevos modelos de sociedad estimulen al niño para la consciente de que no hay realidades por imitar sino futuros por construir, y en el cual las mejores condiciones de vida que se vayan alcanzando exigirán no tanto tener más sino ser más, pues ésta es la verdadera condición del progreso humano.

\section{Metodología de aprendizaje}

La definición de aprendizaje que deben lograr los niños en la educación preescolar, es siempre un aspecto de primer orden en toda sociedad, pues se trata, de establecer aquello que se considera esencial para la preservación, desarrollo de los individuos y del colectivo humano; es un proceso complejo de negociación que sucede en todos los ámbitos educativos, desde el ambiente familiar hasta la institucionalidad educativa, pasando por los contextos comunitarios. En este proceso el niño juega un papel activo, donde sus intereses y necesidades se ponen en manifiesto los intereses sociales, culturales, políticos, económicos del entorno en el que habita, colocándose permanentemente en discusión la relevancia de los aprendizajes que es preciso promover y desarrollar desde la infancia.

Los avances en ciencia, tecnología los procesos de globalización que han ampliado de manera extraordinaria el intercambio humano, el flujo de información, con ello el conocimiento, los valores, creencias, estilos de vida, ideales humanos, sociales, han 


\section{Esther Patricia Suaza-Atencio}

transformado los procesos de socialización. Repercutiendo en los desarrollos cognitivos, afectivos, comunicativos, especialmente en los niños y niñas, ofreciendo nuevos contenidos frente a los que el sujeto, su familia y la sociedad le consolidan. Esta última representada especialmente en el sistema educativo, donde deben ser esencial la pregunta por aquello que es relevante aprender, especialmente en los primeros años de vida.

Para concretar la idea, es preciso explicitar brevemente el concepto de aprendizaje que según Papalia, (1998) las diversas teorías que se han interesado en el aprendizaje coinciden en considerar que se trata de un proceso en el que los seres humanos cambian sus ideas, acciones, comportamiento, actitudes que influye directamente en el desarrollo, el cual a su vez es condición y posibilidad del aprendizaje; se establece por tanto una estrecha reciprocidad entre aprendizaje y desarrollo.

Los aprendizajes son entonces elementos clave para la vida y desarrollo de los sujetos. Los valores, creencias imperantes son determinados por la cultura, aunado a la sociedad, según el entorno. Esta es la razón que impulsa a promover los aprendizajes la búsqueda de un desarrollo integral, significativo para el sujeto y su entorno. La teoría sociocultural asume el aprendizaje y el desarrollo del ser humano como el resultante de las interacciones que suceden en contextos históricos, sociales y culturales.

Como afirmó Vygotsky (1979), en el desarrollo cultural del niño toda función aparece dos veces: primero entre personas (de manera interpsicológica) y después, en el interior del propio niño (de manera interpsicológica). Esto puede aplicarse igualmente a la atención voluntaria, a la memoria lógica y a la formación de conceptos. De acuerdo con el autor, todo aprendizaje tiene su historia previa, puesto que el conocimiento se construye de forma social, es decir, entre personas que interactúan.

De la misma manera, Caputo y Gamallo (2010), expresan que el nivel inicial constituye la primera etapa básica para la incorporación de información del niño o niña en un nuevo medio social diferente al familiar. En ese momento de su 


\section{Esther Patricia Suaza-Atencio}

evolución, el niño o niña interactúa con el medio de formas diversas; cuanto mayor y más variada es esta actividad, más se desarrollan sus capacidades. La gran capacidad de aprendizaje del niño o niña en estas edades está determinada fundamentalmente por dos características importantes; su plasticidad neuronal y el intenso desarrollo de sus órganos sensoriales.

Dicho de otro modo, el aprendizaje relevante moviliza, potencia de manera integral el desarrollo humano, sustentándose en las características, intereses, potencialidades y derechos de cada niño o niña, en coherencia con la movilización y potenciación del desarrollo colectivo. Este criterio de relevancia integra al niño y a la niña como individuos, pero al mismo tiempo los vincula a su realidad social.

Tal como lo certifican, Zapata y Ceballos (2010), la educación en la primera infancia no puede estar basada en actividades mecánicas, repetitivas sin sentido que solo buscan la transmisión unidireccional (del adulto al niño-niña) de información, contenidos poco significativos y motivantes. Por esta razón, los términos educación inicial o educación para la primera infancia provienen de una concepción amplia, que pretende superar esa visión restringida de la educación asociada a la escolarización, que busca llenar de sentido la vida cotidiana de los niños y niñas, apasionarse por su desarrollo como seres humanos y promover su libre expresión y construcción de aprendizajes.

Es propicio destacar que el proceso de aprendizaje no puede orientarse sólo a dotar de herramientas cognitivas o técnicas, no puede limitarse a fines instrumentales. EI aprendizaje es también fundamento, es criterio de orientación de sentido donde el ser humano, en este caso el niño o niña desde sus primeros años, descubre cómo encausar su potencial, como proyectar su ser en relación con otros, qué transformar, qué conservar, y cómo ser feliz reconociéndose uno con los demás y con el entorno.

\section{Principios curriculares}

El principio mediante el cual los docentes propician la didáctica el niño para que construya conocimientos, se encuentra consigo mismo y desarrolla habilidades de comunicación Expresan los logros formativos y de aprendizaje que se espera 


\section{Esther Patricia Suaza-Atencio}

adquieran los niños y niñas en el nivel preescolar. Álvarez, (2011) los define como el conjunto de criterios, planes de estudio, programa, metodologías y procesos que contribuyen a la formación integral y a la construcción de la identidad. Es una manera eficiente y eficaz de organizar para dar cuenta del por qué, para qué con qué, cuándo, y cómo se logran las metas didácticas en el servicio educativo formal del nivel; es la guía del maestro para la práctica educativa en el aula.

Debe incorporar de manera coherente el modelo pedagógico que oriente la práctica el autor García, (2007) indica que la cotidianidad en los procesos de enseñaraprender, se encuentra presente desde la planeación hasta la evaluación, pasando por la ejecución en el aula, centrados en procesos pedagógicos como la integralidad, participación y en lo lúdico.

\section{Integralidad}

La primera infancia es el periodo propicio para potenciar las capacidades cognitivas, comunicativas y sociales. El desarrollo educativo en esta etapa influye en un mejor desempeño en las fases posteriores de la educación, en una disminución del fracaso escolar; en consecuencia, en una reducción de la deserción académica. La concepción que hoy se tiene de la educación para niños y niñas antes de los seis años es concebida como preparación para la escuela (aprestamiento) se caracteriza por prácticas escolares convencionales que privilegian actividades sedentarias, de repetición y de memoria.

El principio de integralidad para Arancibia, (2009) reconoce el trabajo pedagógico que considera al educando como ser único, social en interdependencia y reciprocidad permanente con su entorno familiar, natural, social, étnico y cultural. Plantear el principio de integralidad en el preescolar implica que toda acción educativa debe abarcar las dimensiones del desarrollo del niño, lo socio-afectivo, espiritual, ético, cognitivo, comunicativo, corporal, estético, para potencializarlas y alcanzar niveles de humanización necesarios para su desenvolvimiento en sociedad como un ser humano digno, pleno, autónomo y libre.

Para lograr un desarrollo integral de los niños, es necesario, en los primeros años de 


\section{Esther Patricia Suaza-Atencio}

vida, contar con una apropiada nutrición, atención en salud, amor, estimulación psicosocial e interacciones significativas con sus padres y con otros adultos que ejercen algún tipo de influencia en su proceso de crianza. La educación preescolar, además de reafirmar los procesos de socialización, desarrollo que los niños y las niñas traen de su casa, los introduce al mundo escolar, de la misma manera les crea condiciones para continuar en él, potenciando sus capacidades que les faciliten el aprendizaje escolar y el desarrollo de todas sus dimensiones como seres humanos.

Por tanto, plantea Rosner (2009) que se deben orientar a la solución de problemas abiertos y complejos, como las complejas situaciones que los niños encuentran y resuelven en los contextos naturales relacionados con su mundo físico, afectivo, cognitivo, social, cultural, con una clara intencionalidad pedagógica y didáctica. El niño como ser integral debe ser atendido por otros sectores que tienen competencias específicas, facilitándoles el aprendizaje escolar y el desarrollo de todas sus dimensiones, como también orientándolos a la solución de problemas abiertos y complejos con una clara intencionalidad pedagógica y didáctica.

\section{Participación}

El principio de participación para Martínez (2007) refiere en la educación, la organización y el trabajo de grupo como espacio propicio para la aceptación de sí mismo, del otro, en el intercambio de experiencias, aportes, conocimientos e ideales por parte de los educandos, de los docentes, de la familia y demás miembros de la comunidad a la que pertenece, para la cohesión, el trabajo grupal, la construcción de valores, normas sociales, el sentido de pertenencia y el compromiso grupal y personal.

En el desarrollo del principio de participación se hace relación con la concepción que se tiene de vinculación activa, consciente y permanente de la familia, la sociedad con el Estado, como comunidad educativa, con el objeto de garantizar a los niños, niñas su desarrollo armónico e integral, el ejercicio pleno de sus derechos. Se parte de reconocer que la familia, cualquiera que sea su tipología, es el núcleo primario en el cual los niños han iniciado sus procesos de comunicación, socialización y 
participación; al igual que ha sido el espacio en el que se han construido los primeros vínculos, relaciones afectivas, significaciones hacia sí mismo y hacia los otros.

Este principio reconoce la organización, el trabajo de grupo como espacio propicio para la aceptación de sí mismo, en el intercambio de experiencias, aportes, conocimientos e ideales por parte de los educandos, de los docentes, de la familia y demás miembros de la comunidad a la que pertenece. Se parte de reconocer que la familia, es el núcleo primario en el cual los niños han iniciado sus procesos de comunicación, socialización y participación.

\section{Lúdica}

El juego como dinamizador de la vida del educando mediante el cual se construye conocimientos, se encuentra consigo mismo, con el mundo físico y social, desarrolla iniciativas propias, comparte sus intereses, desarrolla habilidades de comunicación, construye se apropia de normas. Asimismo, Linares y Arias (2016) reconoce que el gozo, entusiasmo, el placer de crear, recrear de generar significados, afectos, visiones de futuro, nuevas formas de acción y convivencia, deben constituir el centro de toda acción realizada por y para el educando, en sus entornos familiar, natural, social, étnico, cultural y escolar.

Para desarrollar el principio de la lúdica Uribe, Taborda y Vélez (2017) expresan que se debe reconocer que el niño es un ser lúdico, esto es, que en lo que él realmente está interesado es en realizar actividades que le produzcan goce, placer y posibilidades de disfrute. El niño es un ser sensible, recién llegado al mundo adulto que trae consigo sus sentimientos, pensamientos, necesita ser tenido en cuenta, querido y cuidado.

Necesita descubrir e intercomunicar sus emociones, sus creencias, las nociones que tiene de las cosas en un clima de confianza, porque de esta manera puede madurar emocionalmente, conocerse, vivir sana, creativa y felizmente. Esto se logra en la medida en que le sea posible recrearse, desarrollar su imaginación e intuición, liberar, reconocer su expresividad, desarrollar habilidades, intercambiar sus puntos 


\section{Esther Patricia Suaza-Atencio}

de vista, reconocer, apreciar su patrimonio cultural, conocer su historia.

Se considera que una persona que se desenvuelve en estas condiciones crece haciéndose presente, activo y creativo en el tejido social, no siendo agresor y menos aún violento. El juego es la expresión máxima del carácter lúdico del niño, para él, el juego se constituye en una actividad fundamental

Todos los niños para Palacio (2016) les gusta jugar, ya que ello les proporciona enorme alegría, a través de él se incorporan a la vida social, al trabajo en equipo, amplían, precisan, construyen conocimientos, forman valores y actitudes. Se puede decir que el juego es una auténtica actividad creadora y colectiva, que produce una profunda satisfacción a los que en ella participan. Se trata de la inmensa alegría de crear, inventar, comunicar y transformar.

Para desarrollar el principio de la lúdica se debe reconocer que el niño es un ser lúdico, esto es, que en lo que él realmente está interesado es en realizar actividades que le produzcan goce, placer y posibilidades de disfrute proporcionándoles enorme alegría, ayudándoles a incorporarse a la vida social, a construir conocimientos y a formar valores y actitudes.

\section{Desarrollo de la dimensión estética}

La actividad en el desarrollo se concibe como la interacción que el sujeto establece con los objetos del mundo físico y social; pasa de un plano externo, sensorial , práctico a uno interno, reflexivo, que le permite encontrar las relaciones que existen entre ellos, representarlas, operar mentalmente para así construir o reconstruir conocimientos, logrando alcanzar de esta forma niveles cada vez más superiores en sus propias, personales formas de pensar de relacionarse con los objetos y las personas.

De allí que en palabras de Castillo (2016), brinda la posibilidad de construir la capacidad humana de sentir, conmoverse, valorar y transformar las percepciones con respecto a si mismo y al entorno, desplegando todas sus posibilidades de acción. El niño al tener interacción con la persona que lo rodea especialmente con sus compañeros, manifiesta sentimientos y emociones, desarrolla la imaginación y el 


\section{Esther Patricia Suaza-Atencio}

gusto estético garantizando climas de confianza y respeto. Desde el punto de vista filosófico la dimensión estética se refiere a la belleza, entendida esta no como lo que dicta la moda, esto es, referida al gusto, sino en sus dos componentes, la verdad y el bien, lo que la enlaza con el aprendizaje desde el propio interior de la verdad y la bondad como paso necesario a las acciones dirigidas hacia el bien personal y el de los demás, que son las bases de la solidaridad.

\section{Acciones generadas en el proceso educativo}

La comprensión de la actividad como forma esencial mediante la cual el niño aprende, logra su desarrollo fundamenta la concepción de una pedagogía activa sobre la cual deben construirse las estrategias a través de las cuales se cumple el proceso pedagógico en el nivel de preescolar.

La pedagogía activa concibe la educación como el señalar caminos para la autodeterminación personal y social, asi como el desarrollo de la conciencia crítica por medio del análisis la transformación de la realidad; acentúa el carácter activo del niño en el proceso de aprendizaje, interpretándolo como buscar significados, criticar, inventar, indagar en contacto permanente con la realidad; concede importancia a la motivación del niño con relación a la escuela-comunidad y vida; identifica al docente como animador, orientador y catalizador del proceso de aprendizaje; concibe la verdad como proyecto que es elaborado y no posesión de unas pocas personas; la relación teoría así como práctica, es un procesos complementarios, y la relación docente-alumno como un proceso de diálogo, cooperación, apertura permanente.

\section{La sensibilidad en el contexto del desarrollo de la dimensión estética}

Desde el ámbito educativo hay que tratar de desarrollar la sensibilidad estética y creativa, mediante todos los recursos expresivos y en todas las facetas de la vida, utilizando los diferentes medios de expresión: lenguaje verbal, musical, plástico, corporal con el fin de poder comunicar sus opiniones, sentimientos y deseos. Villada (2006) indica que de esta manera se desarrolla progresivamente la sensibilidad estética y la capacidad creativa y expresiva, para que aprenda a valorar y disfrutar 


\section{Esther Patricia Suaza-Atencio}

de las obras y manifestaciones artísticas.

Se ubica en el campo de las actitudes, la autoexpresión, el placer y la creatividad que encierra un compromiso, entrega, gratuidad y no obligatoriedad. Hay una estrecha relación entre la sensibilidad, la evolución de la construcción de la autoconciencia, hablar de la sensibilidad es hablar de respuesta pronta ante lo nuevo, de la delicadeza y sutileza, de ofrecer posibilidades de expresión, sentimiento, valoración que permitan al niño su desarrollo en esta dimensión para ser capaz de amarse a sí mismo y amar a los demás, favoreciendo de esta manera el desarrollo de actitudes de pertenencia, autorregulación, confianza, singularidad, eficiencia, satisfacción al lograr lo que a sí mismo se ha propuesto.

La sensibilidad entonces, es hacer referencia a la expresión espontánea que hace el niño de sus emociones, sentimientos, sin que éstos sean prejuzgados, en un clima de seguridad y confianza. Se relaciona con su subjetividad y forma de ver las cosas y se expresa a través del pensamiento mágico-simbólico utilizando los esquemas de pensamiento típicos en el establecimiento de relaciones de semejanzas, diferencias, simbolizaciones, analogías, metáforas, alegorías, paráfrasis, de acuerdo con el nivel de desarrollo y con su propio contexto.

\section{METODOLOGÍA}

Dentro del área de estudio es necesario que se perfilen los pasos que han de darse, en función de alcanzar metas que especifiquen nuevos horizontes de conocimientos. Por ello, en esta fase se desarrollaron ciertos aspectos metodológicos que fundamentan la estructura de la investigación propuesta. De allí que, la utilidad del estudio desde la epistemología reside en igualar el recorrido que permita encontrar los conocimientos fiables con los cuales se realicen aportes imprescindibles al entendimiento de los métodos de aprendizaje para el desarrollo de la dimensión estética en estudiantes de educación inicial

Dentro de este contexto, la metodología cualitativa dispone de métodos sensibles y adecuados para la investigación de una realidad, en donde cada unidad requiere del uso de una lógica no deductiva ni inductiva, sino de una lógica dialéctica, en la cual 
las partes son comprendidas desde el punto de vista del todo, y este a su vez se modifica y enriquece con la comprensión de aquellas.

Para la presente se utilizó el método fenomenológico, el cual, según lo planteado por Ricoeur, citado por Valdés (2008), es un método de descripción de las articulaciones fundamentales de la experiencia, sea esta perceptiva, imaginativa, intelectiva, volitiva, axiológica. Permite además, ver a la reducción fenomenológica como aplicación de la actitud natural, la cual es abandonada cuando se adopta una actitud reflexiva. La misma resulta volver a uno mismo con claridad intelectual y responsabilidad moral junto al deseo de una transparencia absoluta, que haría de la conciencia de sí un saber indudable.

Cuando una investigación se desarrolla desde la fenomenología como método, el investigador queda atrapado en un movimiento infinito de interrogación hacia atrás, con el propósito de definirlo Lebenswelt. Husserl (1992) llega a puntualizar a la fenomenología como el estudio de las estructuras de la conciencia. Este estudio requiere reflexionar acerca de los contenidos de la mente y mediante ellos comprobó una serie de actos como el recordar, desear, percibir e incluso el contenido abstracto de estos actos, a los que llamó significados.

\section{REFLEXIONES FINALES}

Al hablar de lo que facilita el aprendizaje del niño, puede decirse que es la utilización de técnicas fundadas en la experiencia, involucrando activamente al estudiante. Es decir, favorecer y estimular el aprendizaje significativo, entendido este como la interiorización de la experiencia vivida, que provoca en los estudiantes un cambio ligado a tres tipos de conocimientos:

- el saber (conocimiento)

- el saber hacer (habilidad)

- el saber ser (actitud)

Para que se produzca una interiorización de la experiencia de aprendizaje, es necesario que ella tenga un sentido particular para el individuo. Al respecto, Mezirow (2010) ha reflexionado acerca de la manera como los estudiantes construyen, 


\section{Esther Patricia Suaza-Atencio}

validan y reformulan el sentido de sus experiencias a partir de sus aprendizajes. El niño aprende, principalmente, cuando da un sentido a su aprendizaje y puede también aportar cambios en su vida a causa de ellos.

En este contexto es importante destacar el método del aprendizaje experiencial, el cual se basa en la corriente humanista, tiene como fin el desarrollo de la persona que aprende. Hemos dicho que el niño debe aprender a lo largo de toda su vida y que debe ser un estudiante activo, basando su proceso de aprendizaje en la acción. En consecuencia, la pedagogía experiencial favorece la puesta en práctica de los nuevos aprendizajes a partir de un ajuste personal indispensable para pasar de la teoría a la práctica.

El aprendizaje experiencial tiene su origen en las ideas de Dewey (1967), quien establece que el aprendizaje es un proceso que integra la experiencia y la teoría; donde la experiencia da vida a la teoría y la teoría le da sentido a la experiencia.

Por su parte Kolb (1977), propone un modelo estructural del aprendizaje bajo la forma de un ciclo de aprendizaje compuesto por cuatro etapas:

- Experiencia concreta

- Observación reflexiva

- Conceptualización abstracta

- Experimentación activa

Estas cuatro etapas son las que conducen a una conceptualización abstracta que será transferida a la situación real. El niño experimenta constantemente con sus conceptos y los modifica como consecuencia de sus observaciones y experiencias. Asimismo, Kolb (1977) considera el aprendizaje como un proceso que permite al ser humano deducir conceptos y principios a partir de su experiencia, para orientar su conducta en situaciones nuevas, y modificar esos conceptos incrementando su eficacia.

Por esta razón, cualquiera que sean los métodos o técnicas a utilizar, estas deben favorecer que el niño se involucre en su experiencia de aprendizaje: es él quien debe observar, probar, analizar, participar en las distintas actividades del proceso para integrar los nuevos conocimientos y desarrollar la imaginación, el gusto estético 


\section{Esther Patricia Suaza-Atencio}

garantizando climas de confianza y respeto, donde los lenguajes artísticos se expresan y juegan un papel fundamental al transformar lo contemplado en metáforas y representaciones armónicas de acuerdo con las significaciones propias de su entorno natural, social y cultural..

\section{REFERENCIAS CONSULTADAS}

1. Álvarez, C. (2011). Tesis doctoral: La relación teoría-práctica en la enseñanza y el desarrollo profesional docente. Un estudio de caso en Educación. Educere, vol. 19, núm. 63, pp. 363-371. Universidad de los Andes. Mérida, Venezuela.

2. Zapata, B. E. \& Ceballos, L. (2010). Opinión sobre el rol y perfil del educador para la primera infancia. Revista Latinoamericana de Ciencias Sociales, Niñez y Juventud, 8 (2), pp. 1069-1082.

3. Arancibia, V. (2009). La educación de alumnos con talentos: una deuda y una oportunidad para Chile. Santiago de Chile: Pontificia Universidad Católica de Chile.

4. Caputo, M. \& Gamallo, G. (2010). Infancia, desarrollo y conocimiento: los niños y niñas y su socialización. Revista Latinoamericana de Ciencias Sociales, Niñez y Juventud. 8 (2), pp. 849-860.

5. Dewey, J. (1967). Experiencia y Educación. Buenos Aires: Losada.

6. García Pérez, F. F. (2007). El profesorado y el conocimiento: una relación compleja. En Portada, 63, pp. 24-26.

7. Husserl, Edmund. (1992) Ideas related to a Pure Phenomenology and a Phenomenological Philosophy. FCE México.

8. Kolb, D. (1977). Aprendizaje y solución de problemas. En D. Kolb, I. Rubin \& J. Mclntyre (Eds.), Psicología de las organizaciones: problemas contemporáneos. Madrid: Prentice/May

9. Linares, A. \& Arias, L. (2016). Estrategias lúdicas para hacer del tiempo libre un espacio de formación en valores. Institución Universitaria Los Libertadores.

10. Martínez Sánchez, J. A. (2007) "La dirección escolar, ¿una cuestión sin resolver?", Participación Educativa, 5 (Se puede ver en 


\section{Esther Patricia Suaza-Atencio}

http://www.mepsyd.es/cesces/revista/n5-martinez-sanchez.pdf, consultado el 20 de febrero de 2010). MEC (1978). Educación

11. Mezirow, J. (2010). Learning as transformation. Critical perspectives on theory in progress. San Francisco, CA., EE. UU.: Jossey-Bass.

12. Organización para la Cooperación y el Desarrollo Económico. OCDE (2017) El trabajo de la OCDE sobre educación y competencias. Disponible en: https://www.oecd.org/education/El-trabajo-de-la-ocde-sobre-educacion-ycompetencias.pdf

13. Palacio, M. (2016). Descansos escolares: momentos lúdicos en la Institución Educativa Vida para Todos. Trabajo de grado para optar al título de especialista en pedagogía de la lúdica en la Universidad los Libertadores. Recuperado de http://repository.libertadores.edu.co/handle/11371/448.

14. Papalia, D. (1998). Psicología del desarrollo. Buenos Aires: McGraw-Hill.

15. Ricoeur, P (1992) Introduction to 'Ideas I' by Edmundo Husserl. Translation and notes by Raúl Velozo from the book Idées guidelines pour une phénoménologie. Gallimard Paris.

16. Rosner, W. (2009,). Estándares de calidad en la formación de profesores de alumnos con altas capacidades. Retrieved from http://tvaustral.uach.cl/Play.aspx?video=A2D90D85592F50A4DEDAD7C6702 AD.

17. Uribe, L.; Taborda, A., \& Vélez, A. (2017). Los espacios lúdicos, como estrategia pedagógica, para fortalecer la sana convivencia, en el descanso escolar. Fundación Universitaria Los Libertadores., Medellín, Colombia.

18. Villada. (2006). La expresión corporal en la formación inicial del profesorado. Estudio y análisis de los Curricular de las titulaciones de magisterio. Tesis Doctoral. Universidad de Oviedo.

19. Vygotsky, L. (1978). Mind in society. Cambridge, MA.: Harvard University Press. 


\section{REFERENCES CONSULTED}

1. Álvarez, C. (2011). Doctoral thesis: The theory-practice relationship in teaching and teaching professional development. A case study in Education. Educere, vol. 19, no. 63, pp. 363-371. University of the Andes. Merida, Venezuela. 2. Zapata, B.

2. E. \& Ceballos, L. (2010). Opinion on the role and profile of the educator for early childhood. Latin American Journal of Social Sciences, Children and Youth, 8 (2), pp. 1069-1082.

3. Arancibia, V. (2009). The education of talented students: a debt and an opportunity for Chile. Santiago de Chile: Pontifical Catholic University of Chile.

4. Caputo, M. \& Gamallo, G. (2010). Children, development and knowledge: children and their socialization. Latin American Journal of Social Sciences, Children and Youth. 8 (2), pp. 849-860.

5. Dewey, J. (1967). Experience and Education Buenos Aires: Losada.

6. García Pérez, F. F. (2007). Teachers and knowledge: a complex relationship. In Portada, 63, pp. 24-26.

7. Husserl, Edmund. (1992) Ideas related to a Pure Phenomenology and a Phenomenological Philosophy. FCE Mexico.

8. Kolb, D. (1977). Learning and problem solving. In D. Kolb, I. Rubin \& J. Mclntyre (Eds.), Psychology of organizations: contemporary problems. Madrid: Prentice / May

9. Linares, A. \& Arias, L. (2016). Playful strategies to make leisure time a space for values training. Los Libertadores University Institution.

10. Martínez Sánchez, JA (2007) "School management, an unresolved issue?", Educational Participation, 5 (Can be seen at http://www.mepsyd.es/cesces/revista/n5-martinez-sanchez .pdf, accessed February 20, 2010). MEC (1978). Education

11. Mezirow, J. (2010). Learning as transformation. Critical perspectives on theory in progress. San Francisco, CA., USA UU .: Jossey-Bass.

12. Organization for Economic Cooperation and Development. OECD (2017) The work of the OECD on education and skills. Available at: https://www.oecd.org/education/El-trabajo-de-la-ocde-sobre-educacion-ycompetencias.pdf 
13. Palacio, M. (2016). School breaks: playful moments at the Vida para Todos Educational Institution. Degree work to apply for the title of specialist in playful pedagogy at Los Libertadores University. Recovered from http://repository.libertadores.edu.co/handle/11371/448.

14.Papalia, D. (1998). Developmental Psychology Buenos Aires: McGraw-Hill.

15. Ricoeur, P (1992) Introduction to 'Ideas I' by Edmundo Husserl. Translation and notes by Raúl Velozo from the book Idées guidelines pour une phénoménologie. Gallimard Paris

16. Rosner, W. (2009,). Quality standards in the training of teachers of students with high capacities. Retrieved from http://tvaustral.uach.cl/Play.aspx?video=A2D90D85592F50A4DEDAD7C6702 AD.

17. Uribe, L .; Taborda, A., \& Vélez, A. (2017). Playful spaces, as a pedagogical strategy, to strengthen healthy living together, during school break. Los Libertadores University Foundation., Medellín, Colombia.

18. Villada. (2006). Body expression in initial teacher training. Study and analysis of the Curricula of the teaching qualifications. Doctoral thesis. Oviedo University.

19. Vygotsky, L. (1978). Mind in society Cambridge, MA .: Harvard University Press. 